\title{
Graded index photonic hole: Analytical and rigorous full wave solution
}

\author{
Shiyang Liu, ${ }^{1,2}$ Li Li, ${ }^{2,3}$ Zhifang Lin, ${ }^{2,3,4}$ H. Y. Chen, ${ }^{5,4}$ Jian Zi, ${ }^{3,2}$ and C. T. Chan ${ }^{4}$ \\ ${ }^{1}$ Institute of Information Optics, Zhejiang Normal University, Jinhua, Zhejiang 321004, China \\ ${ }^{2}$ Surface Physics Laboratory and Department of Physics, Fudan University, Shanghai 200433, China \\ ${ }^{3}$ Key Laboratory of Micro and Nano Photonic Structures (Ministry of Education), Fudan University, Shanghai, China \\ ${ }^{4}$ Physics Department, Hong Kong University of Science and Technology, Clear Water Bay, Kowloon, Hong Kong \\ ${ }^{5}$ School of Physical Science and Technology, Soochow University, Suzhou, Jiangsu 215006, China
}

(Received 3 August 2010; published 27 August 2010)

\begin{abstract}
We present a rigorous full wave approach to the omnidirectional photonic hole $(\mathrm{PH})$, an optical system inspired by celestial phenomena and characterized by a radially graded refractive index $n(r) \sim 1 / r^{\alpha / 2}$. It is analytically demonstrated that light capture is effective for $\alpha \geq \alpha_{c}=2$. Our analyses are corroborated by precise numerical simulations of steady-state and time-evolution behaviors. The simulations indicate that the optical energy entering the PH system can be kept in captivity for a time duration $t_{d}$ scaling as $t_{d} \sim 1 /\left(\alpha_{c}-\alpha\right)$ when $\alpha$ approaches $\alpha_{c}$. A crucial difference in the time evolution of fields is shown between the cases with $\alpha$ close to and equal to $\alpha_{c}$.
\end{abstract}

DOI: 10.1103/PhysRevB.82.054204

PACS number(s): 42.25.-p, 41.20.Jb

Nature has been a source of inspiration to many scientific and technological advances. For a long time, the natureinspired designs of optical systems are predominantly based on mimicking structures appearing in the diversity of the biological world, leading to a thriving area of biomimetic or bioinspired photonics. ${ }^{1-4}$ Typical examples in this area include, among others, the study of structural $\operatorname{color}^{5-7}$ and artificial compound eyes. ${ }^{8}$

In addition to imitating terrestially occurring structures, the design of novel optical components is recently inspired by mimicking celestial phenomena. Interesting examples are optical attractors and photon traps that mimic celestial mechanics. ${ }^{9-11}$ These optical analogs of celestial phenomena may find niche applications in light control, optoelectronics, and solar-energy harvesting. Their fabrications are enabled by the advances of nanofabrication techniques and transformation optics. ${ }^{12,13}$ The former offers a wide range of possibilities to locally tailor the electromagnetic (EM) response $^{14-16}$ while the latter allows the transmutation of dielectric singularities into a manageable topological defect. $^{17,18}$

One intriguing astroinspired optical device is motivated by the black hole, a fascinating celestial phenomenon. It is characterized essentially by a radially graded refractive index $n(r) \sim 1 / r^{\alpha / 2} \cdot{ }^{10,11,19}$ In contrast to the bioinspired photonics that directly solves Maxwell's equations, the astroinspired optical design starts with the study of ray trajectories. In the ray tracing limit, it is shown for integer power index (PI) $\alpha$ that $\alpha \geq 2$ constitutes the condition for the ray trajectories to fall into the core of the system, resulting in an optical attractor (OA) or "photonic black hole," $10,11,19$ in the sense that all light satisfying certain criterions will be held in captivity inside the system. However, the critical PI $\alpha_{c}$ for light trapping has not yet been identified under arbitrary wavelength conditions, and interesting phenomena occurring when $\alpha$ comes (close) to $\alpha_{c}$ remain unexplored.

In this paper, we present the first rigorous full wave analysis in the astroinspired photonics ${ }^{9-11}$ for the photonic hole (PH) system. Starting with an arbitrary real $\alpha$, the critical PI $\alpha_{c}=2$ for the capture of light is derived analytically based on wave optics as well as ray optics. At $\alpha=\alpha_{c}$, a criterion is established for light trapping. The analytical analyses are corroborated, and also, intriguing phenomena when $\alpha$ approaches and reaches $\alpha_{c}$ are explored, by precise numerical simulations in both the frequency domain (FD) and the time domain (TD).

Consider a nonmagnetic system with a radially graded refractive index ${ }^{19}$

$$
n(r)= \begin{cases}\left(\frac{r_{s}}{r}\right)^{\alpha / 2}, & r \leq r_{s}, \\ 1, & r>r_{s},\end{cases}
$$

where $r_{s}$ denotes the radius of the $\mathrm{PH}$ system. The ray trajectory is then governed by ${ }^{20}$

$$
\frac{d r}{d \theta}=-\frac{r}{q} \sqrt{n^{2} r^{2}-q^{2}},
$$

where $(r, \theta)$ are polar coordinates and $q=r_{s} \sin \theta_{s} \leq r_{s}$ denotes the distance between the incident ray (before entering the $\mathrm{PH}$ ) and the center of the $\mathrm{PH}$ system. Equations (1) and (2) yield the ray trajectories inside the $\mathrm{PH}$

$$
r= \begin{cases}r_{s}\left[\frac{\sin \theta_{s}}{\sin \left(\theta-\frac{\alpha}{2} \theta+\frac{\alpha}{2} \theta_{s}\right)}\right]^{\gamma}, & \alpha \neq 2, \\ r_{s} e^{-\left(\theta-\theta_{s}\right) \cot \theta_{s}}, & \alpha=2\end{cases}
$$

with $\gamma=2 /(2-\alpha)$. For $\alpha<2$, an incident ray with $q \neq 0$ can never reach the center of the system. ${ }^{17}$ Instead, it escapes after arriving at the closest point $r_{\min }=r_{s} \sin ^{\gamma} \theta_{s}$ away from the center. For $\alpha \geq 2$, on the other hand, it follows from Eq. (3) that the rays spiral into the PH center. So $\alpha_{c}=2$ is identified as the critical PI for light trapping in the limit of ray optics.

We next concentrate on the rigorous full wave analysis. We illustrate the approach in two dimensions (2D) for the transverse magnetic (TM) modes with the electric $(E)$ field parallel to the axis of the cylindrical PH. Generalization to 
other polarization modes and three dimensions is straightforward. The $n(r)$ profile in Eq. (1) allows an analytical solution, suggesting that the $E$ field for $r<r_{s}$

$$
E_{\mathrm{int}}= \begin{cases}\sum_{m}\left[b_{m} J_{\nu}(\rho)+c_{m} H_{\nu}^{+}(\rho)\right] e^{i m \theta}, & \alpha<2, \\ \sum_{m}\left[b_{m} r^{\beta}+c_{m} r^{-\beta}\right] e^{i m \theta}, & \alpha=2, \\ \sum_{m}\left[b_{m} H_{\nu}^{+}(\rho)+c_{m} H_{\nu}^{-}(\rho)\right] e^{i m \theta}, & \alpha>2,\end{cases}
$$

where $b_{m}$ and $c_{m}$ are constant coefficients, $J_{\nu}(\rho)$ and $H_{\nu}^{+}(\rho)\left[H_{\nu}^{-}(\rho)\right]$ are the Bessel function and Hankel function of the first (second) kind, respectively, and

$$
\begin{gathered}
\beta= \begin{cases}\sqrt{m^{2}-x_{s}^{2}}, & |m|>x_{s}, \\
-i \sqrt{x_{s}^{2}-m^{2}}, & |m|<x_{s},\end{cases} \\
\nu=\left|\frac{2 m}{2-\alpha}\right|, \quad \rho=\frac{2 k_{0} r}{|2-\alpha|}\left(\frac{r_{s}}{r}\right)^{\alpha / 2}
\end{gathered}
$$

with $x_{s}=k_{0} r_{s}$ and $k_{0}$ being the wave number in the outside medium. The time dependence of $e^{-i \omega t}$ is omitted for brevity. Any incident wave can be expanded in terms of partial waves (PWs) $J_{m}\left(k_{0} r\right) e^{i m \theta}$ of different angular momentum indices $m$ as

$$
E_{\mathrm{inc}}=\sum_{m} p_{m} J_{m}\left(k_{0} r\right) e^{i m \theta}
$$

with $p_{m}$ the PW expansion coefficients.

Define

$$
P=-\int_{0}^{2 \pi}\langle\boldsymbol{S}\rangle \cdot \boldsymbol{e}_{r} r_{c} d \theta, \quad U=\int_{r_{c}}^{r_{s}} \int_{0}^{2 \pi}\langle u\rangle r d \theta d r,
$$

where $\langle\boldsymbol{S}\rangle$ and $\langle u\rangle$ are the time-averaged Poynting vector and energy density, respectively, $\boldsymbol{e}_{r}$ denotes the radial unit vector, and $r_{c}$ is a tiny quantity. To establish a physical steady state in the FD, $\langle u\rangle$ must be integrable, implying a vanishing $P$ and a finite $U$ in the limit of $r_{c} \rightarrow 0 .^{21}$ The PH effect manifests itself if and only if no integrable steady-state solution (ISS) can be reached in the FD. With Eq. (4), $P$ and the dominant part of $U$ as $r_{c} \rightarrow 0 \mathrm{read}$

$$
\begin{gathered}
P=\frac{(2-\alpha)}{k_{0}} \sum_{m}\left[\left|c_{m}\right|^{2}+\operatorname{Re}\left(b_{m} c_{m}^{*}\right)\right], \\
U \sim\left|c_{0}\right|^{2} \ln r_{c}+\sum_{m \neq 0}\left|c_{m}\right|^{2} r_{c}^{-2|m|}
\end{gathered}
$$

for $\alpha<2$,

$$
P= \begin{cases}-\frac{2 \pi}{k_{0}} \sum_{m} \beta \operatorname{Im}\left(b_{m} c_{m}^{*}\right), & \text { if }|m|>x_{s}, \\ \frac{\pi}{k_{0}} \sum_{m}|\beta|\left(\left|b_{m}\right|^{2}-\left|c_{m}\right|^{2}\right), & \text { if }|m|<x_{s},\end{cases}
$$

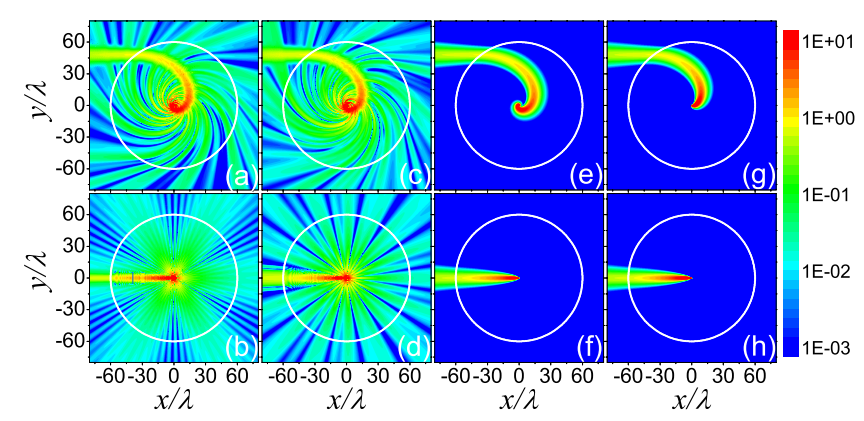

FIG. 1. (Color online) Magnetic field intensity patterns of a Gaussian beam incident on the PH systems of $r_{s}=60 \lambda$ and [(a) and (b)] $\alpha=1.99$, [(c) and (d)] 1.999, [(e) and (f)] 2, and [(g) and (h)] 2.5. The white circles outline the boundary of the system. The beam has a waist radius $w_{0}=4 \lambda$ and is focused at $(0,48 \lambda)$ and $(0,0)$ for upper and lower panels, respectively. Here $\lambda$ is the wavelength of the beam in the outer medium. For $\alpha \geq 2$, the field pattern is not a physical ISS (unless a tiny absorption is introduced), it is a demonstration of the PH effect.

$$
U \sim \begin{cases}\sum_{m} \beta^{-1} m^{2}\left|c_{m}\right|^{2} r_{c}^{-\beta}, & \text { if }|m|>x_{s}, \\ \sum_{m}\left(\left|c_{m}\right|^{2}+\left|b_{m}\right|^{2}\right) \ln r_{c}, & \text { if }|m|<x_{s}\end{cases}
$$

for $\alpha=2$, and

$$
\begin{aligned}
& P=\frac{(\alpha-2)}{k_{0}} \sum_{m}\left[\left|b_{m}\right|^{2}-\left|c_{m}\right|^{2}\right], \\
& U \sim \sum_{m}\left(\left|b_{m}\right|^{2}+\left|c_{m}\right|^{2}\right) r_{c}^{-(\alpha-2) / 2}
\end{aligned}
$$

for $\alpha>2$. Equation (8) implies that an ISS in the FD can be achieved for $\alpha<2$ provided that $c_{m}=0$, consistent with the Mie theory for a homogeneous scatter, where $c_{m}=0$ is imposed ad hoc to avoid field divergence at $r=0$. For $\alpha=2$, one must distinguish between incident PWs with $|m|>x_{s}$ and $|m|<x_{s}$. For the PWs with $|m|>x_{s}$, an ISS can still be reached with $c_{m}=0$ while for those with $|m|<x_{s}$, Eq. (9) suggests that $\langle u\rangle$ is no longer integrable, indicating that PWs with an angular momentum small enough will be attracted to the $\mathrm{PH}$ center, resulting in a logarithmic singularity in the EM energy. It is therefore concluded that the critical PI is $\alpha_{c}=2$ from the full-wave analysis. In addition, $c_{m}$ must vanish to ensure inward energy flux $(P \geq 0)$, as required by causality. Finally, for $\alpha>2$, Eq. (10) reveals that no ISS exists, and $c_{m}=0$ should be imposed again by causality $P \geq 0$. We have solved the problem for a system where $n(r)$ is given by Eq. (1) except that a tiny core with $n=n_{c}$ for $r \leq r_{c}$ is introduced. Our solution justifies the argument $c_{m}=0$ in the limit of $r_{c} \rightarrow 0$, irrespective of the value of $\alpha .^{22}$

To corroborate the analyses above, we have performed precise full wave numerical simulations for various values of $\alpha$ based on the generalized Mie theory (see Appendix A). Typical results are shown in Fig. 1 for a Gaussian beam incident on the PH systems with $\alpha=1.99,1.999,2$, and 2.5. For $\alpha<2$, the beam first spirals toward the central region, then escapes out of the $\mathrm{PH}$, resulting in an outward winding 


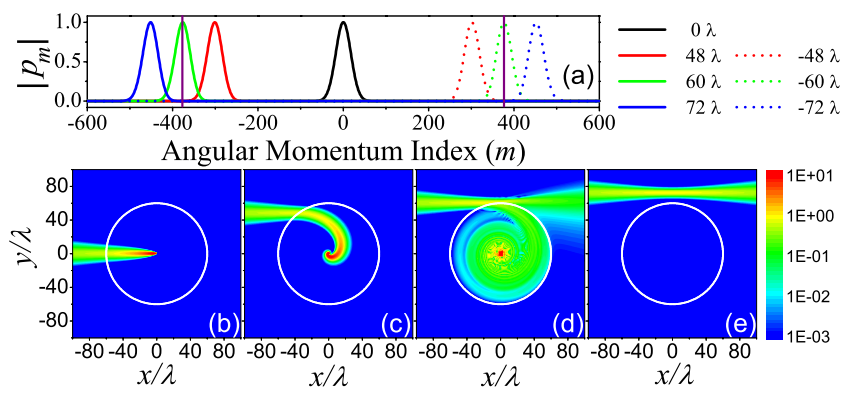

FIG. 2. (Color online) (a) $\left|p_{m}\right|$ versus angular-momentum index $m$ for a Gaussian beam with waist radius $w_{0}=4 \lambda$ and focused at $(0$, $0),(0, \pm 48 \lambda),(0, \pm 60 \lambda)$, and $(0, \pm 72 \lambda)$. Two vertical solid lines mark the position of $\pm x_{s}$. The corresponding magnetic field intensity patterns are shown in (b), (c), (d), and (e) for beam focused at $(0,0),(0,48 \lambda),(0,60 \lambda)$, and $(0,72 \lambda)$, respectively. The system has $r_{s}=60 \lambda$ and $\alpha=\alpha_{c}$.

field pattern, as shown in Figs. 1(a) and 1(c). From Figs. 1(b) and 1(d), the incident beam is seen to spread out even when it is focused on the $\mathrm{PH}$ center. This, arising from the wave nature of light, is in contrast to the ray optics analysis that assumes vanishing ray widths [see Eq. (3) for ray trajectories]. As $\alpha$ increases from 1.999 to 2, the field pattern undergoes a radical change, strongly reminiscent of a phase transition in statistical physics. The beam exhibits a spiral trajectory toward the core, as displayed in Figs. 1(e)-1(g). Consequently, the full-wave simulations confirm $\alpha_{c}=2$. Besides, as $\alpha$ increases further, the beam becomes less spiral, as illustrated by a comparison between Figs. 1(e) and 1(g). It is emphasized that no commercially available software that employs a finite-sized grid can reproduce the transitionlike field patterns as $\alpha$ tends to $\alpha_{c}$.

The "critical point" $\alpha_{c}$ exhibits unique phenomena that deserve more attention. Based on Eq. (9), a PH effect manifests itself only for PWs with $|m|<x_{s}$. This is typically exhibited in Fig. 2. For an incident beam focused at the $\mathrm{PH}$ center, the distribution of the PWs is centered around $m=0$ and the beam is trapped. As the axis of the incident beam moves away from the $\mathrm{PH}$ center, the distribution of PWs shifts away from $m=0$. If the beam axis is not far away from the PH center, it still falls into the PH core since all its PWs with significant amplitudes lie within $|m|<x_{s}$, as illustrated in Figs. 2(a)-2(c). When the beam axis moves further away from the PH center, a fraction of its PWs goes outside $|m|$ $<x_{s}$, then only part of the beam spirals toward the center, whereas the rest escapes, leading to a split of beam. This is shown in Fig. 2(d), a case corresponding to the green solid curve located almost symmetrically around the vertical line in Fig. 2(a). For a beam with all its PWs of significant amplitudes falling beyond $|m|<x_{s}$, the system has no visually observable effect on it, as displayed in Fig. 2(e), a case corresponding to the blue solid curve on the left hand side of the vertical line in Fig. 2(a).

For systems like this, where the ISS may not exist, it is very important to study the transient processes. We exemplify the study by simulating the time-evolution behavior of an optical pulse excited by a line source $1.5 r_{s}$ away from the PH of $r_{s}=60 \lambda$, as schematically plotted in Fig. 3 . The pulse has a Gaussian temporal profile

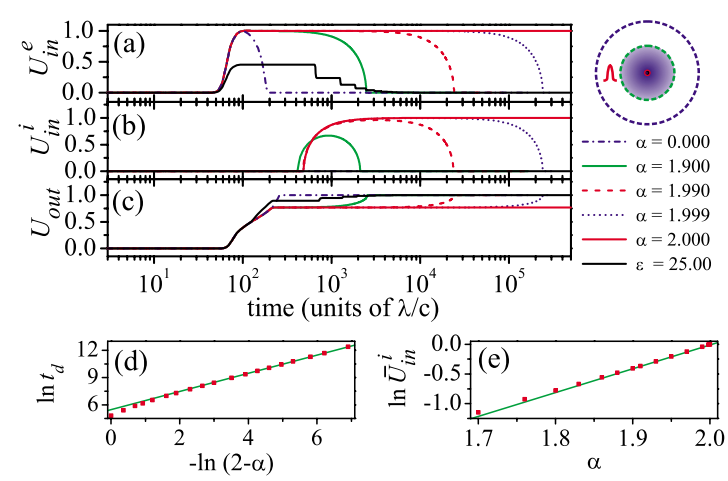

FIG. 3. (Color online) (a)-(c) The normalized EM energy versus time. $U_{i n}^{e}, U_{i n}^{i}$, and $U_{\text {out }}$ are, respectively, the normalized EM energy inside the $\mathrm{PH}$, that inside the core of the $\mathrm{PH}$, and that radiated into infinity. They are evaluated by integrating the Poynting vector at enclosures marked by the green, red, and blue circles shown schematically in the upper-right corner. Both $U_{i n}^{i}(t)$ and $U_{i n}^{e}(t)$ are normalized to $\max \left\{U_{\text {in }}^{e}(t)\right\}$ for $\alpha=0$ while $U_{\text {out }}(t)$ is normalized to $\lim U_{\text {out }}(t)$ for $\alpha=0$. With the increase of $\alpha$, the corresponding lines in (a)-(c) appear from left hand side to right hand side in sequence, while for $\epsilon=25$, it is a stairlike solid line. The logarithmic of the time duration $t_{d}$ for the pulse energy being kept inside the system is plotted versus $-\ln (2-\alpha)$ in (d). The logarithmic of the maximal energy $\bar{U}_{i n}^{i}$ accumulated in the core of radius $r_{c}=0.05 \lambda$ is plotted versus $\alpha$ in (e). The green straight lines in (d) and (e), serving as guides to eyes, have slopes 1 and 4 , respectively. $U_{i n}^{e}$ for a rod with $\varepsilon=n^{2}=25$ has a stair-case like decay behavior because of the sharp discontinuity in wave impedance.

$$
E_{\mathrm{inc}}(r, t)=\int_{-\infty}^{+\infty} A(\omega) H_{0}^{+}\left(k_{0}\left|\boldsymbol{r}-\boldsymbol{r}_{0}\right|\right) e^{-i \omega t} d \omega
$$

where $A(\omega)=\tau /(2 \sqrt{\pi}) e^{-\tau^{2}\left(\omega-\omega_{0}\right)^{2} / 4}$ and $\omega_{0}=2 \pi c / \lambda$ with $c$ being the speed of light in vacuum. Figures 3 and 4 exhibit the typical results with spectral width $2 / \tau=\omega_{0} / 20$ and various values of $\alpha$. The normalized EM energy $U_{i n}^{e}$ inside the PH is displayed versus time in Fig. 3(a), which shows that, for $\alpha$ $<2, U_{i n}^{e}$ decays to zero after reaching its maximum. This implies that the energy entering the system will completely escape eventually, although a system with a larger $\alpha$ will hold the energy inside for a longer time. While for $\alpha=2$, the energy going into the system is trapped, at least within the time limit of our simulations, as shown by the red solid line

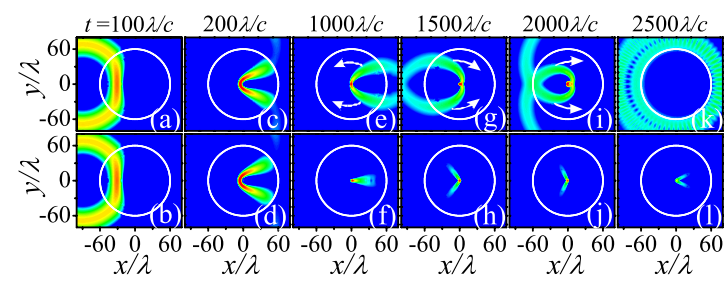

FIG. 4. (Color online) Magnetic field intensity patterns at some typical time stages when the PH is illuminated with a pulse excited by a line source. The upper row and the lower row correspond to the cases with $\alpha=1.9$ and $\alpha=2$, respectively. The white arrows denote the directions of rotation for two light branches. 
in Fig. 3(a). To describe how long the pulse energy stays in the system, we define $t_{s}(\alpha)$ as the time at which $U_{i n}^{e}$ decays to vanishing. Then $t_{d}=t_{s}(\alpha)-t_{s}(0)$ characterizes the time duration for the energy being kept in captivity. In Fig. 3(d), we plot $\ln t_{d}$ versus $-\ln (2-\alpha)$. The linear relation shown for $\alpha$ close to 2 indicates a scaling law $t_{d} \sim 1 /(2-\alpha)$ and confirms $\alpha_{c}=2$.

The scaling behavior $t_{d} \sim 1 /\left(\alpha_{c}-\alpha\right)$ can also be worked out based on ray optics. From the ray trajectory Eq. (3), it follows that for $\alpha<2$, a ray at a distance $q=r_{s} \sin \theta_{s}<r_{s}$ away from the $\mathrm{PH}$ center will spiral toward the central region of the $\mathrm{PH}$, until it reaches the closest point at $(r, \theta)$ $=\left(r_{\min }, \theta_{\min }\right)$ with $r_{\min }=r_{s} \sin ^{\gamma} \theta_{s}$ and $\theta_{\min }=(\pi-\alpha \theta) /(2-\alpha)$, then it escapes out of the PH by a symmetric trajectory. As a result, the light energy will be kept in captivity for a duration of $t_{d}$ given by

$$
t_{d}=2 \int_{\theta_{s}}^{\theta_{\min }} \frac{\sqrt{(d r / d \theta)^{2}+r^{2}}}{c / n(r)} d \theta .
$$

Using Eqs. (1)-(3), one obtains the scaling law

$$
t_{d}=\frac{r_{s}}{c} \frac{4 \cos \theta_{s}}{2-\alpha} \sim \frac{1}{\alpha_{c}-\alpha}, \quad \text { for } \theta_{s} \neq 0 .
$$

In Fig. 3(b), we plot the normalized EM energy $U_{i n}^{i}$ inside a tiny core of radius $r_{c}=0.05 \lambda$ versus time. A comparison between Figs. 3(a) and 3(b) indicates that the light energy enters the core region before escaping out of the system, in agreement with the FD demonstration in Fig. 1. In addition, as $\alpha$ increases, the maximum energy $\bar{U}_{i n}^{i}$ accumulated in the core increases. In Fig. 3(e) we display $\ln \bar{U}_{i n}^{i}$ versus $\alpha$. The linear relation implies $\bar{U}_{i n}^{i} \sim e^{4(\alpha-2)}$. For $\alpha=2$, both $U_{i n}^{e}$ and $U_{i n}^{i}$ tend to unity, suggesting that all energy entering the system is finally converged to the core. The normalized energy radiated into infinity $U_{\text {out }}$ versus time is presented in Fig. 3(c). For $\alpha<2, U_{\text {out }}$ tends to 1 , indicating that all the pulse energy will be radiated to infinity eventually. While for $\alpha$ $=2, U_{\text {out }}<1$, confirming that part of the pulse energy is trapped in the $\mathrm{PH}$.

The time evolution of fields is rather intriguing, especially when $\alpha$ is close to $\alpha_{c}$. Figure 4 shows a series of field patterns at some typical stages of time for $\alpha=1.9$ and $\alpha=\alpha_{c}$ $=2$. The pulse excited by a line source is seen to enter the PH and turn into two branches with no significant difference between $\alpha=1.9$ and $\alpha=2$ at earlier time, as shown in Figs. 4(a)-4(d). In the subsequent period, the two light branches rotate in opposite directions due to nonvanishing angularmomentum components, as shown in Figs. 4(e)-4(j). For $\alpha$ $=1.9$, the energy keeps leaking out of the system as the light wings spin around the center. While for $\alpha=2$, no visually observable leakage occurs as the two wings of light converge spirally toward the center. Eventually, for $\alpha=1.9$, all light energy escapes from the system, even for those once arrived close to the system center whereas for $\alpha=2$, all light energy spirals toward center, as illustrated in Figs. 4(k) and 4(1) [see also Figs. 3(a) and 3(b)].

In summary, we have developed a rigorous full wave approach to the $\mathrm{PH}$, a typical optical system in the astroinspired photonics. The light capture for $\alpha \geq \alpha_{c}=2$ is demonstrated analytically and corroborated by precise numerical simulations in both the FD and the TD. For $\alpha=\alpha_{c}$, a criterion is established that the light trapping is effective for incident PWs satisfying $|m|<x_{s}$ for TM modes. The TD simulations indicate that the optical energy entering the $\mathrm{PH}$ system is kept inside for a time duration $t_{d}$ scaling as $t_{d} \sim 1 /\left(\alpha_{c}-\alpha\right)$ when $\alpha$ approaches $\alpha_{c}$. The maximum EM energy that can be accumulated in core region of the system obeys $\bar{U}_{i n}^{i}$ $\sim e^{4\left(\alpha-\alpha_{c}\right)}$, though this energy will eventually escape for $\alpha$ $<\alpha_{c}$. Finally, our simulations show a crucial difference in the time evolution of fields between $\alpha$ close to and equal to $\alpha_{c}$.

Finally, it is noted that the basic physics discussed here applies to other waves. More discussion on the practical applications and possible experimental realization of the $\mathrm{PH}$ and its analogs in acoustic and linear liquid surface waves can be found in the Appendices B and C.

This work is supported by the China 973 Program, NNSFC (10774028, 10904020, J0730310), MOE of China (Grant No. B06011), SSTC (Grant No. 08dj1400302), and CPSF (Grant No. 200902211). Work at HK is supported by HKRGC through GRF under Grant No. 600209.

\section{APPENDIX A: GENERALIZED MIE THEORY FOR CYLINDRICAL PHOTONIC HOLE}

In this appendix, we give mathematical formulations of the generalized Mie theory for the cylindrical photonic hole (PH) system with the refractive index $n$ given by Eq. (1), recapitulated below for convenience

$$
n(r)=\left\{\begin{array}{cc}
\left(\frac{r_{s}}{r}\right)^{\alpha / 2}, & r \leq r_{s}, \\
1, & r>r_{s} .
\end{array}\right.
$$

The electric field inside the PH for the TM modes (with the electric field along $z$ direction) is given by

$$
E_{\mathrm{int}}= \begin{cases}\sum_{m=-\infty}^{+\infty} b_{m} J_{\nu}(\rho) e^{i m \theta}, & \alpha<2, \\ \sum_{m=-\infty}^{+\infty} b_{m} r^{\beta} e^{i m \theta}, & \alpha=2, \\ \sum_{m=-\infty}^{+\infty} b_{m} H_{\nu}^{+}(\rho) e^{i m \theta}, & \alpha>2,\end{cases}
$$

where $(r, \theta)$ denote 2D polar coordinates, $b_{m}$ are constant coefficients whereas $c_{m}$ in Eq. (4) have been set to zero based on the arguments given in the text. $J_{\nu}(\rho)$ and $H_{\nu}^{+}(\rho)$ are the Bessel function and the Hankel function of the first kind, respectively, and

$$
\beta= \begin{cases}\sqrt{m^{2}-x_{s}^{2}}, & |m|>x_{s}, \\ -i \sqrt{x_{s}^{2}-m^{2}}, & |m|<x_{s},\end{cases}
$$




$$
\nu=\left|\frac{2 m}{2-\alpha}\right|, \quad \rho=\frac{2 k_{0} r}{|2-\alpha|}\left(\frac{r_{s}}{r}\right)^{\alpha / 2}
$$

with $x_{s}=k_{0} r_{s}$. The incident and scattered fields read, respectively,

$$
E_{\mathrm{inc}}=\sum_{m=-\infty}^{+\infty} p_{m} J_{m}\left(k_{0} r\right) e^{i m \theta}
$$

and

$$
E_{s}=-\sum_{m=-\infty}^{+\infty} a_{m} H^{+}\left(k_{0} r\right) e^{i m \theta}
$$

Matching the boundary conditions at $r=r_{s}$ yields the Mie scattering coefficients

$$
\begin{aligned}
& \frac{a_{m}}{p_{m}}= \begin{cases}\frac{J_{\nu}\left(\rho_{s}\right) J_{m}^{\prime}\left(x_{s}\right)-J_{m}\left(x_{s}\right) J_{\nu}^{\prime}\left(\rho_{s}\right)}{J_{\nu}\left(\rho_{s}\right) H_{m}^{\prime}\left(x_{s}\right)-H_{m}^{+}\left(x_{s}\right) J_{\nu}^{\prime}\left(\rho_{s}\right)}, & \alpha<2, \\
\frac{x_{s} J_{m}^{\prime}\left(x_{s}\right)-\beta J_{m}\left(x_{s}\right)}{x_{s} H_{m}^{+\prime}\left(x_{s}\right)-\beta H_{m}^{+}\left(x_{s}\right)}, & \alpha=2, \\
\frac{H_{\nu}^{+}\left(\rho_{s}\right) J_{m}^{\prime}\left(x_{s}\right)+J_{m}\left(x_{s}\right) H_{\nu}^{+\prime}\left(\rho_{s}\right)}{H_{\nu}^{+}\left(\rho_{s}\right) H_{m}^{\prime}\left(x_{s}\right)+H_{m}^{+}\left(x_{s}\right) H_{\nu}^{+\prime}\left(\rho_{s}\right)}, & \alpha>2,\end{cases} \\
& \frac{b_{m}}{p_{m}}= \begin{cases}\frac{2 i /\left(\pi x_{s}\right)}{J_{\nu}\left(\rho_{s}\right) H_{m}^{\prime}\left(x_{s}\right)-H_{m}^{+}\left(x_{s}\right) J_{\nu}^{\prime}\left(\rho_{s}\right)}, & \alpha<2, \\
\frac{2 i / \pi}{r_{s}^{\beta}\left[x_{s} H_{m}^{+\prime}\left(x_{s}\right)-\beta H_{m}^{+}\left(x_{s}\right)\right]}, & \alpha=2, \\
\frac{2 i /\left(\pi x_{s}\right)}{H_{\nu}^{+}\left(\rho_{s}\right) H_{m}^{\prime}\left(x_{s}\right)+H_{m}^{+}\left(x_{s}\right) H_{\nu}^{+\prime}\left(\rho_{s}\right)}, & \alpha>2,\end{cases}
\end{aligned}
$$

where the primes denote the derivative with respect to argument and $\rho_{s}=2 x_{s} /|2-\alpha|$. With the use of $\boldsymbol{H}=\nabla \times \boldsymbol{E} /\left(i \omega \mu_{0}\right)$, the magnetic field inside the PH system can be worked out as $\boldsymbol{H}_{\text {int }}=Z\left(H_{r} \boldsymbol{e}_{r}+H_{\theta} \boldsymbol{e}_{\theta}\right)$, where $\boldsymbol{e}_{r}$ and $\boldsymbol{e}_{\theta}$ are unit vectors in radial and polar directions, respectively, $Z$ is the wave impedance of the outside medium,

$$
H_{r}= \begin{cases}\sum_{m=-\infty}^{+\infty} b_{m} \frac{m}{k_{0} r} J_{\nu}(\rho) e^{i m \theta}, & \alpha<2, \\ \sum_{m=-\infty}^{+\infty} b_{m} \frac{m}{k_{0} r} r^{\beta} e^{i m \theta}, & \alpha=2, \\ \sum_{m=-\infty}^{+\infty} b_{m} \frac{m}{k_{0} r} H_{\nu}^{+}(\rho) e^{i m \theta}, & \alpha>2\end{cases}
$$

and

$$
H_{\theta}= \begin{cases}\sum_{m=-\infty}^{+\infty} i b_{m}\left(\frac{r_{s}}{r}\right)^{\alpha / 2} J_{\nu}^{\prime}(\rho) e^{i m \theta}, & \alpha<2, \\ \sum_{m=-\infty}^{+\infty} i b_{m} \frac{\beta}{k_{0} r} r^{\beta} e^{i m \theta}, & \alpha=2, \\ \sum_{m=-\infty}^{+\infty} i b_{m}\left(\frac{r_{s}}{r}\right)^{\alpha / 2} H_{\nu}^{+\prime}(\rho) e^{i m \theta}, & \alpha>2 .\end{cases}
$$

In practical numerical calculation, the summation over $m$ is cutoff at $m_{c}=400$, which guarantees the convergence of all our results in the frequency domain.

Our simulation on the transient processes starts with the rigorous full wave solution in the frequency domain. The data in frequency domain are then transformed to the time domain by the fast Fourier transform. ${ }^{23}$ As $\alpha$ approaches $\alpha_{c}=2$, one needs an increasing number $N_{f}$ of frequency points to guarantee the time-domain resolution and convergence. For example, while $\alpha=1$ requires $N_{f}=2048$ frequency points, $\alpha=1.999$ takes $N_{f}=61440$ frequency points to achieve reliable time-domain resolution.

\section{APPENDIX B: AN EXAMPLE OF APPLICATION}

The photonic hole system can serve as an omnidirectional photonic trap, enabling the confinement of light incident from arbitrary direction for a duration $t_{d}$ scaling like $1 /\left(\alpha_{c}-\alpha\right.$ ) (for $\alpha \lesssim \alpha_{c}$ ), as has been discussed in the text. In this appendix, we illustrate one more example for the practical application of the $\mathrm{PH}$ system. Figure 5 shows the magnetic field intensity patterns when the $\mathrm{PH}$ is illuminated by a line source located 400 $\lambda$ away. It can be seen that for $\alpha=0$, the signal near the origin $(x, y)=(0,0)$ may be too weak for a sensor to detect, as shown in Fig. 5(a). If we introduce a PH system, the magnetic field near the origin will be significantly enhanced, due to the light concentrating characteristics of the PH system, as exhibited in Figs. 5(b)-5(f). As a result, by placing the sensor near the $\mathrm{PH}$ center, the signal detection will be greatly facilitated. It is noted that the field enhancement near the PH center does not require $\alpha \geq 2$. The case with $\alpha=1.5$ will be good enough, as displayed in Fig. 5(c), where the magnetic field intensity (for steady state) near the origin is enhanced by a factor of 50. For time dynamical case, we show in Fig. $6 P, U$, and $P_{1}$ versus time when a pulse is excited by a line source $400 \lambda$ away from the $\mathrm{PH}$ system. Here $P, U$, and $P_{1}$ are defined by

$$
P(t)=-\int_{0}^{2 \pi} \boldsymbol{S} \cdot \boldsymbol{e}_{r} r_{c} d \theta, \quad U(t)=\int P(t) d t
$$

and

$$
P_{1}(t)=-\int_{0}^{2 \pi} \boldsymbol{S} \cdot \boldsymbol{e}_{r} r_{c} \theta d \theta
$$

with $\boldsymbol{e}_{r}$ denoting the radial unit vector and $S$ the timedependent Poynting vector. The pulse has a Gaussian temporal profile given by Eq. (11) in the text and a spectral width $\omega_{0} / 20$. By comparing $P$ or $U$ versus time for $\alpha \geq 1$ with that 


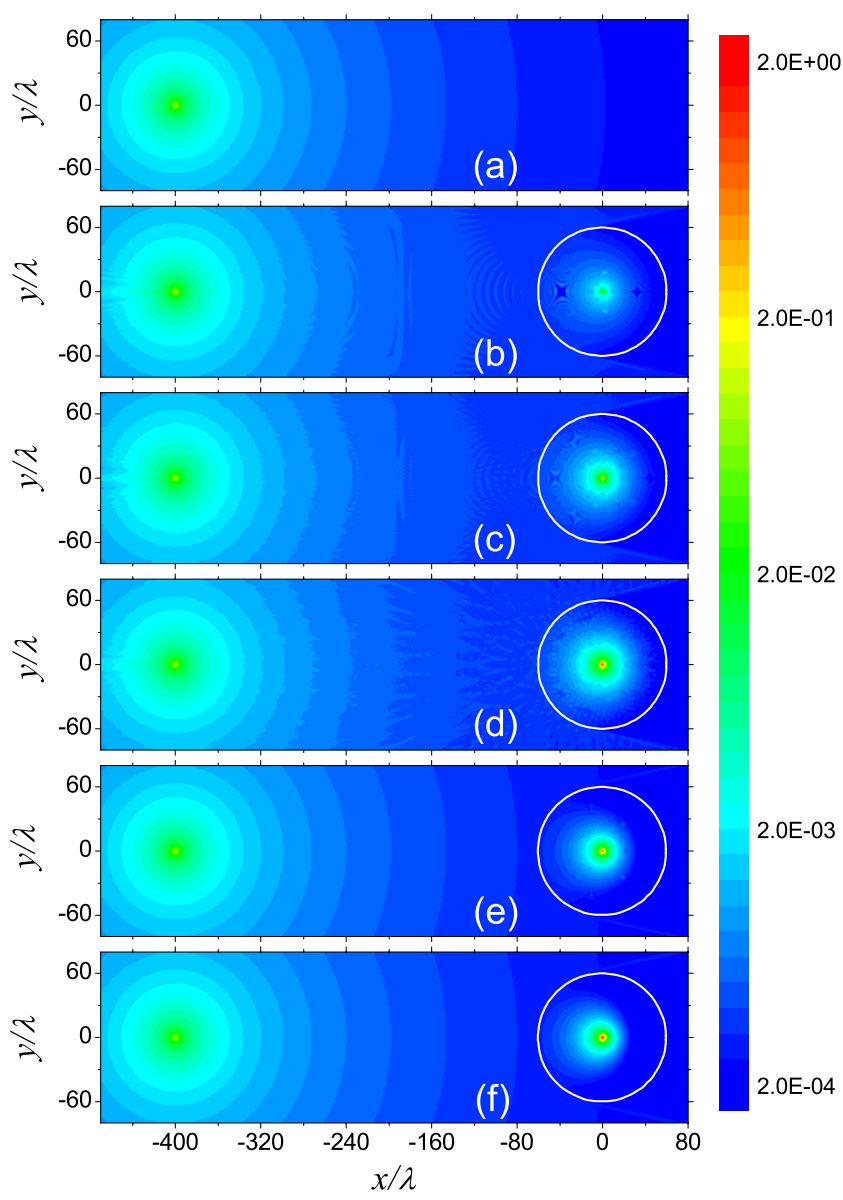

FIG. 5. (Color online) Magnetic field intensity patterns when a line source is placed 400 $\lambda$ away from the PH system with $r_{s}=60 \lambda$ and (a) $\alpha=0$, (b) 1.0, (c) 1.5, (d) 1.9, (e) 2.0, and (f) 2.5. The white circles outline the boundaries of the PH systems with $\alpha \geq 1$. The field intensity is considerably enhanced near the PH center, suggesting the signal detection may be facilitated by putting a sensor near the $\mathrm{PH}$ center.

for $\alpha=0$, it is clearly seen that the sensitivity will be significantly enhanced if the sensor is enclosed by a PH system. In addition, due to the feature of omnidirectionality of the $\mathrm{PH}$ system, the ratio $P_{1} / P$ determines the direction from which the signal comes. As a result, with two sensors, each enclosed by a PH system, the location of the signal source can be detected, as schematically plotted in Fig. 6(d).

\section{APPENDIX C: POSSIBLE MEANS OF EXPERIMENTAL REALIZATION AND ANALOGS IN OTHER WAVES}

The radially graded refractive index for the $\mathrm{PH}$ system may be implemented by using EM metamaterials, ${ }^{11}$ as the EM invisibility cloaks. ${ }^{24}$ The main difficulty may lie in the dielectric singularity. This difficulty actually arises also in invisibility cloaks, where the difficulty of dielectric singularity is further aggravated by the extreme anisotropy. As EM cloaks of various forms have been demonstrated from microwave to near optical frequencies, we may expect a good possibility for the $\mathrm{PH}$ system to be realized. To be more specific, there are at least two possible ways to circumvent
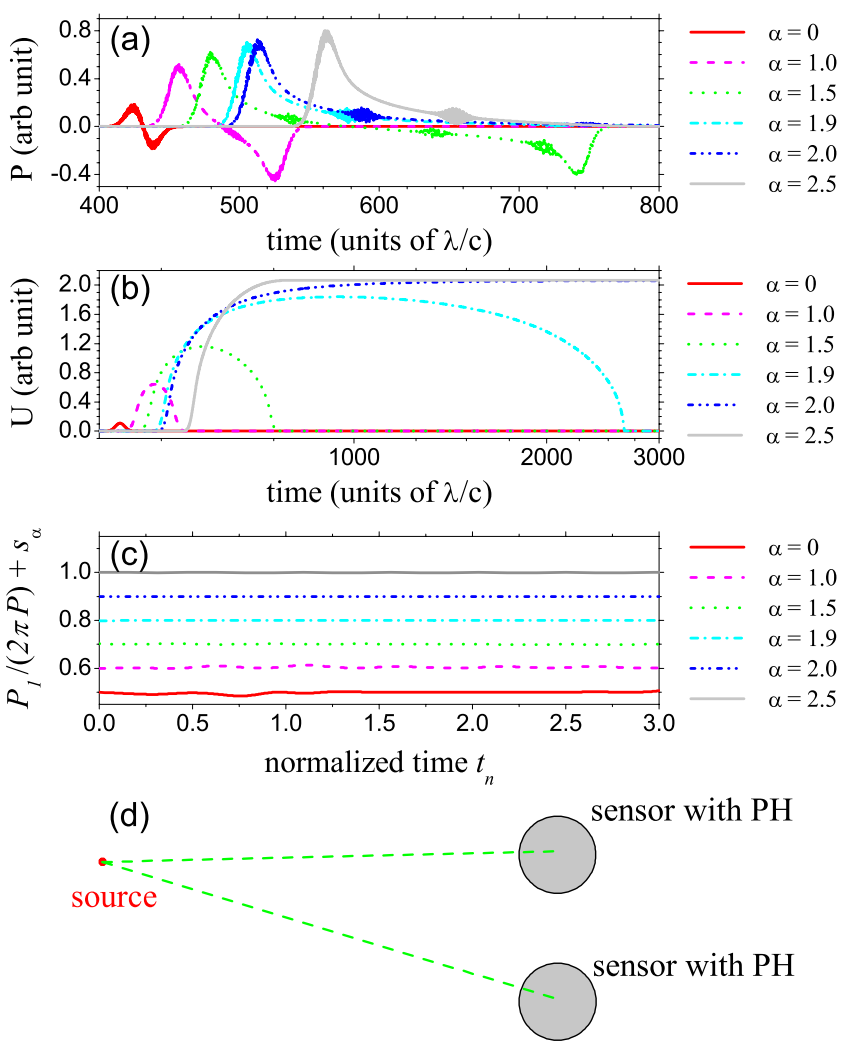

FIG. 6. (Color online) (a) $P$ versus time and (b) $U$ versus time when a pulse of Gaussian temporal profile is excited by a line source $400 \lambda$ away from the PH of $r_{s}=60 \lambda$. The data shown are for $r_{c}=6 \lambda$, see, Eq. (B1). The signal is significantly enhanced due to the light concentrating characteristics of the $\mathrm{PH}$ system, leading to a sensor with enhanced sensitivity. (c) $P_{1} /(2 \pi P)+s_{\alpha}$ versus normalized time $t_{n}$, showing that due to the feature of omnidirectionality, $P_{1} / P$ determines the direction from which the signal comes. Here $s_{\alpha}=0,0.1,0.2,0.3,0.4$, and 0.5 for $\alpha=0.0,1.0,1.5,1.9,2.0$, and 2.5 , respectively. It is introduced for a clear demonstration showing that the signal originated from direction $\theta=180^{\circ}$. The normalized time $t_{n}=1$ corresponds to the time at which $P$ reaches its positive peak. (d) Schematic plot showing how two sensors enclosed by the $\mathrm{PH}$ systems can be used to locate the position of the source.

the difficulty of dielectric singularity, both inherited from the EM cloaks. One possible way is to "transmute" away the dielectric singularities into a mere topological defect using the technique of transformation optics. ${ }^{17}$ This approach works for $\alpha<2$. We note that based on the idea of "transmutation," an omnidirectional retroreflector, which originally requires material parameter with polynomial type of singularity, has been successfully fabricated in experiment. ${ }^{18}$ The other way is to implement the design using a "reduced set of parameters," which means an approximate set of parameters in the field of transformation optics. This is the way by which the invisibility cloak was first realized. ${ }^{24}$ Appropriate truncation near the center of the photonic hole system may be one of the simplest ways of "reduction." We note that a "reduced version" of photonic hole system with $\alpha=2$ has been experimentally realized in microwave frequencies, ${ }^{25}$ suggesting a possibility that the photonic hole-type metamaterials can indeed be realized. 

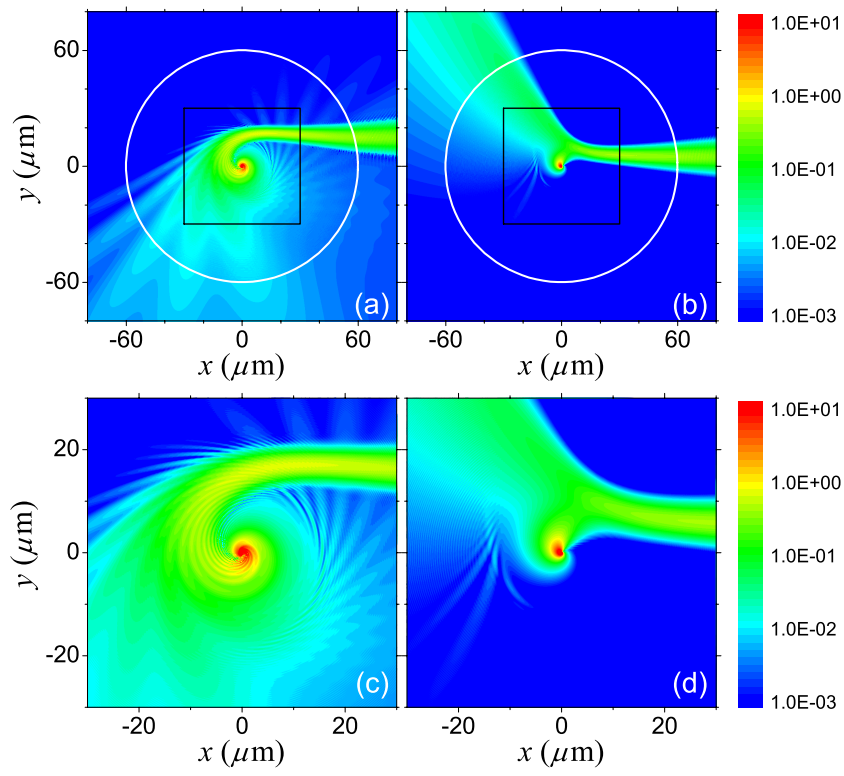

FIG. 7. (Color online) Magnetic field intensity pattern when Gaussian beams are incident on the OA systems of $r_{s}=60 \mu \mathrm{m}$. The refractive index is given by Eq. (D1) with $(a, b, c)=(\sqrt{325}$, $-20,1) \mu \mathrm{m}$ for (a) and (c) and $(a, b, c)=(\sqrt{125},-20,1) \mu \mathrm{m}$ for (b) and (d). The white circles outline the boundaries of the OA systems and the black squares in (a) and (b) denote the regions showing in (c) and (d), respectively. The beam has a waist radius $w_{0}=4 \lambda$ and is focused at (a) $(0,15 \mu \mathrm{m})$ and (b) $(0,5 \mu \mathrm{m})$. The incident wavelength is $\lambda=0.5 \mu \mathrm{m}$ in the outer medium. Note that the field patterns are actually not physical integrable steady-state solutions (unless a tiny absorption is introduced), it is a demonstration of the OA effect.

The basic physics discussed in the text applies to other waves, some analogs of the PH system may be implemented in the acoustic waves and/or linear liquid surface waves. For instance, it has long been known that for ( $z$-invariant) 2D case, wave equations for acoustics and electromagnetics are equivalent. When 2D EM wave of transverse magnetic modes (with the electric field $\boldsymbol{E}$ along $z$ direction) maps to acoustic wave, the permittivity $\varepsilon$ corresponds to $\lambda^{-1}$, the reciprocal of fluid bulk modulus. ${ }^{26}$ Fabrication of acoustic metamaterials with a small bulk modulus may be experimentally less challenging, which is expected to enable an acoustic analog of the PH system. In addition, by comparing the shallow-water wave equation ${ }^{27}$

$$
\nabla \cdot(h \nabla \eta)+\frac{\omega^{2}}{g} \eta=0
$$

with the 2D EM wave equation for the transverse electric modes (with the magnetic field $\boldsymbol{H}$ parallel to $z$ direction)

$$
\nabla \cdot\left(\varepsilon^{-1} \nabla H_{z}\right)+\mu \omega^{2} H_{z}=0,
$$

we can map an EM system to a linear liquid surface wave system, provided that $\varepsilon$ is replaced by $h^{-1}, \mu$ by $g^{-1}$, and $H_{z}$ by $\eta$. Here $\eta$ is the vertical displacement of the liquid surface, $g$ is the gravitational acceleration, $h$ is the depth of the water, and $\omega$ is the angular frequency. The depth $h$ of the liquid can be tuned by modulating the bottom with relative ease. As a result, the mapping between $\varepsilon$ and $h^{-1}$ offers a great possibility to realize a liquid surface wave analog of the PH system, which may be expected to find applications in controlling the flow of ocean wave energy. We are working along this line.

\section{APPENDIX D: GENERALIZED MIE THEORY FOR CYLINDRICAL OPTICAL ATTRACTOR}

In this appendix, we present mathematical formulations of the generalized Mie theory for the cylindrical optical attractor $(\mathrm{OA})$ system, ${ }^{11}$ characterized by the refractive index

$$
n(r)= \begin{cases}{\left[\left(\frac{a}{r}\right)^{2}+\frac{b}{r}+c\right]^{1 / 2},} & r \leq r_{s}, \\ 1, & r>r_{s} .\end{cases}
$$

The radially graded refractive index profile (D1) also allows an analytical solution to the wave equation for the electric field of TM modes, suggesting, for $r<r_{s}$,

$$
E_{\mathrm{int}}= \begin{cases}\sum_{m=-\infty}^{+\infty} b_{m} e^{-i \rho_{c r} \beta} M\left(q^{\prime}, q, 2 i \rho_{c}\right) e^{i m \theta}, & c \neq 0, \\ \sum_{m=-\infty}^{+\infty} b_{m} r^{\beta} J_{2 \beta}\left(\rho_{b}\right) e^{i m \theta}, & c=0 \text { and } b \neq 0, \\ \sum_{m=-\infty}^{+\infty} b_{m} r^{\beta} e^{i m \theta}, & b=c=0,\end{cases}
$$

where $M\left(q^{\prime}, q, z\right) \equiv{ }_{1} F_{1}\left(q^{\prime}, q, z\right)$ denotes the Kummer confluent hypergeometric function ${ }^{28}$ (also known as the confluent hypergeometric function of the first kind), and

$$
\begin{gathered}
\beta= \begin{cases}\sqrt{m^{2}-x_{a}^{2}}, & |m|>x_{a}, \\
-i \sqrt{x_{a}^{2}-m^{2}}, & |m|<x_{a},\end{cases} \\
\rho_{c}=\sqrt{c} k_{0} r, \quad \rho_{b}=2 \sqrt{b / r} k_{0} r, \\
q=2 \beta+1, \quad q^{\prime}=\frac{1}{2}\left(q+i k_{0} b / \sqrt{c}\right)
\end{gathered}
$$

with $x_{a}=k_{0} a$. The coefficients $c_{m}$ for another set of mathematical solutions involving the confluent hypergeometric function of the second kind $U\left(q^{\prime}, q, z\right)$ (Ref. 28) or the Hankel function have been set to zero based on the same arguments as given in the text. Note that Bessel functions are actually special cases of the confluent hypergeometric functions ${ }^{28}$ with the relation given by

$$
M(\mu, 2 \mu, 2 i x)=e^{i x}\left(\frac{x}{2}\right)^{1 / 2-\mu} \Gamma\left(\mu+\frac{1}{2}\right) J_{\mu-1 / 2}(x) .
$$

Matching the boundary conditions at $r=r_{s}$ yields the Mie scattering coefficients. The magnetic field $\boldsymbol{H}$ is again determined by $\boldsymbol{H}=\nabla \times \boldsymbol{E} /\left(i \omega \mu_{0}\right)$. In numerical simulation, the confluent hypergeometric functions are evaluated by direct 
summation of Kummer's series using extended precision subroutines. ${ }^{29}$ Figure 7 displays, as examples, the magnetic field intensity pattern when Gaussian beams are incident on the OA systems with $(a, b, c)=(\sqrt{325},-20,1)$ and $(a, b, c)$ $=(\sqrt{125},-20,1)$, corresponding, respectively, to Figs. 1(d) and 1(e) in Ref. 11. The light is seen to be torn apart, with part of the beams spiralling toward the OA center, while the rest scattered into infinity, in agreement with Ref. 11.
${ }^{1}$ G. Zuccarello, D. Scribner, R. Sands, and L. J. Buckley, Adv. Mater. 14, 1261 (2002).

${ }^{2}$ P. Vukusic and J. R. Sambles, Nature (London) 424, 852 (2003).

${ }^{3}$ A. R. Parker and H. Townley, Nat. Nanotechnology 2, 347 (2007).

${ }^{4}$ L. P. Lee and R. Szema, Science 310, 1148 (2005).

${ }^{5}$ J. Zi, X. Yu, Y. Li, X. Hu, C. Xu, X. Wang, X. Liu, and R. Fu, Proc. Natl. Acad. Sci. U.S.A. 100, 12576 (2003).

${ }^{6}$ S. Kinoshita, S. Yoshioka, and J. Miyazaki, Rep. Prog. Phys. 71, 076401 (2008).

${ }^{7}$ H. Kim, J. Ge, J. Kim, S.-e. Choi, H. Lee, H. Lee, W. Park, Y. Yin, and S. Kwon, Nat. Photonics 3, 534 (2009).

${ }^{8}$ K. H. Jeong, J. Kim, and L. P. Lee, Science 312, 557 (2006).

${ }^{9}$ U. Leonhardt and T. G. Philbin, New J. Phys. 8, 247 (2006).

${ }^{10}$ E. E. Narimanov and A. V. Kildishev, Appl. Phys. Lett. 95, 041106 (2009).

${ }^{11}$ D. A. Genov, S. Zhang, and X. Zhang, Nat. Phys. 5, 687 (2009).

${ }^{12}$ J. B. Pendry, D. Schurig, and D. R. Smith, Science 312, 1780 (2006).

${ }^{13}$ U. Leonhardt, Science 312, 1777 (2006).

${ }^{14}$ W. Cai, U. K. Chettiar, A. V. Kildishev, and V. M. Shalaev, Nat. Photonics 1, 224 (2007).

${ }^{15}$ J. Valentine, S. Zhang, T. Zentgraf, E. Ulin-Avila, D. A. Genov, G. Bartal, and X. Zhang, Nature (London) 455, 376 (2008).

${ }^{16}$ J. Valentine, J. Li, T. Zentgraf, G. Bartal, and X. Zhang, Nature Mater. 8, 568 (2009).

${ }^{17}$ T. Tyc and U. Leonhardt, New J. Phys. 10, 115038 (2008).
${ }^{18}$ Y. G. Ma, C. K. Ong, T. Tyc, and U. Leonhardt, Nature Mater. 8, 639 (2009).

${ }^{19} n(r)$ for "photonic black hole" studied in Ref. 11 has the form $\left(\frac{a}{r^{2}}+\frac{b}{r}+c\right)^{1 / 2}$. It allows an analytical solution involving confluent hypergeometric functions (see Appendix D) though it blocks considerably the transparency of physical analysis. Here, we reduce the problem to the most physically transparent form while keeping the essence of the physics.

${ }^{20} \mathrm{M}$. Born and E. Wolf, Principles of Optics, 7th ed. (Cambridge University Press, Cambridge, 1999).

${ }^{21}$ J. van Bladel, Singular Electromagnetic Fields and Sources (Oxford University Press, New York, 1991).

${ }^{22}$ For $\alpha \geq 2$, a tiny absorption is introduced to reach the steadystate solution with $c_{m}=0$ in the limit of $r_{c} \rightarrow 0$.

${ }^{23}$ E. O. Brigham, The Fast Fourier Transform and Its Applications (Prentice-Hall, Eaglewood Cliffs, 1988).

${ }^{24}$ D. Schurig, J. J. Mock, B. J. Justice, S. A. Cummer, J. B. Pendry, A. F. Starr, and D. R. Smith, Science 314, 977 (2006).

${ }^{25}$ Q. Cheng, T. Cui, W. Jiang, and B. Cai, New J. Phys. 12, 063006 (2010).

${ }^{26}$ S. A. Cummer and D. Schurig, New J. Phys. 9, 45 (2007).

${ }^{27}$ See, e.g., M. W. Dingemans, Water Wave Propagation over Uneven Bottoms (World Scientific, Singapore, 1997).

${ }^{28}$ L. J. Slater, Confluent Hypergeometric Functions (Cambridge University Press, Cambridge, 1960).

${ }^{29}$ M. Nardin, W. F. Perger, and A. Bhalla, ACM Trans. Math. Softw. 18, 345 (1992). 